\title{
ON FABER POLYNOMIALS GENERATED BY AN $m$-STAR
}

\author{
J. BARTOLOMEO AND MATTHEW HE
}

\begin{abstract}
In this paper, we study the Faber polynomials $F_{n}(z)$ generated by a regular $m$-star $(m=3,4, \ldots)$

$$
S_{m}=\left\{x \omega^{k} ; 0 \leq x \leq 4^{1 / m}, k=0,1, \ldots, m-1, \omega^{m}=1\right\} .
$$

An explicit and precise expression for $F_{n}(z)$ is obtained by computing the coefficients via a Cauchy integral formula. The location and limiting distribution of zeros of $F_{n}(z)$ are explored. We also find a class of second-order hypergeometric differential equations satisfied by $F_{n}(z)$. Our results extend some classical results of Chebyshev polynomials for a segment $[-2,2]$ in the case when $m=2$.
\end{abstract}

\section{INTRODUCTION}

Let $E$ be a compact set (not a single point) whose complement $\mathbf{C}^{*} \backslash E$ with respect to the extended plane is simply connected. The Riemann mapping theorem asserts that there exists a conformal mapping $w=\Phi(z)$ of $\mathbf{C}^{*} \backslash E$ onto the exterior of a circle $|w|=\rho_{E}$ in the $w$-plane. For a unique choice of $\rho_{E}$, we can insist that

$$
\Phi(\infty)=\infty, \quad \Phi^{\prime}(\infty)=1,
$$

so that, in a neighborhood of infinity,

$$
\Phi(z)=z+a_{0}+\frac{a_{1}}{z}+\frac{a_{2}}{z^{2}}+\cdots .
$$

The polynomial part of $\{\Phi(z)\}^{n}$, denoted by $F_{n}(z)=z^{n}+\cdots$, is called the Faber polynomial of degree $n$ generated by the set $E$.

Let

$$
\Psi(w)=w+b_{0}+\frac{b_{1}}{w}+\frac{b_{2}}{w^{2}}+\cdots
$$

be the inverse function of $w=\Phi(z)$. Thus, $\Psi(w)$ maps the domain $|w|>\rho_{E}$ conformally onto $\mathbf{C}^{*} \backslash E$. Faber [2] proved that

$$
\frac{\Psi^{\prime}(w)}{\Psi(w)-z}=\sum_{n=0}^{\infty} \frac{F_{n}(z)}{w^{n+1}}, \quad|w|>\rho_{E}, \quad z \in E .
$$

The Faber polynomials play an important role in approximation theory and geometric function theory. It can be shown that, under suitable conditions, a

Received by the editor March 20, 1992 and, in revised form, November 17, 1992.

1991 Mathematics Subject Classification. Primary 30C15, 41A58.

Key words and phrases. Faber polynomials, $m$-star, zero distribution. 
function analytic in the inner domain of a Jordan curve $J$ can be expanded into a series of Faber polynomials that come from the mapping function of the outer domain of $J$ (cf. [1]).

The explicit construction of the Faber polynomials of a given set $E$ depends essentially on the knowledge of the mapping function $\Phi(z)$. For $E=[-2,2]$, we know that $\Phi(z)=\left(z+\sqrt{z^{2}-4}\right) / 2$ with inverse $\Phi(w)=w+1 / w$. For $n \geq 1$, the polynomial part of $\{\Phi(z)\}^{n}$ is the same as the polynomial part of

$$
\Phi^{n}(z)+\Phi^{-n}(z)=w^{n}+w^{-n},
$$

which reduces to $2 \cos n \theta$, when $w=e^{i \theta}$. Thus the Faber polynomials are (apart from a multiplicative constant) the same as the classical Chebyshev polynomials $T_{n}(x)$ for $[-2,2]$.

The following properties for $T_{n}(x)$ are well known [7]:

(i) For $n \geq 1$,

$$
T_{n}(x)=n \sum_{j=0}^{\left[\frac{n}{2}\right]}(-1)^{j} \frac{(n-j-1) !}{(n-2 j) ! j !} x^{n-2 j},
$$

where

$$
\left[\frac{n}{2}\right]= \begin{cases}\frac{n}{2} & \text { if } n \text { is even } \\ \frac{n-1}{2} & \text { if } n \text { is odd }\end{cases}
$$

(ii) $T_{n}(x)$ satisfies the following differential equation:

$$
\left(4-x^{2}\right) y^{\prime \prime}-x y^{\prime}+n^{2} y=0 .
$$

(iii) The zeros of $T_{n}(x)$ are located on $(-2,2)$ for every $n \geq 1$.

(iv) The asymptotic behavior of the zeros of $T_{n}(x)$ is given by the arcsine distribution

$$
d \mu(t)=\frac{1}{\pi} \frac{1}{\sqrt{4-t^{2}}} d t .
$$

In this paper we shall study the Faber polynomials generated by a regular $m$-star $(m=2,3, \ldots)$

$$
S_{m}=\left\{x \omega^{k} ; 0 \leq x \leq 4^{1 / m}, k=0,1, \ldots, m-1, \omega^{m}=1\right\} .
$$

Clearly, $S_{m}$ becomes $[-2,2]$ when $m=2$. It is natural to ask what can be said about Faber polynomials when $m=3,4,5, \ldots$. By using the properties and characteristics of the Chebyshev polynomials as a motivation, we are able to compute an explicit representation for $F_{n}(z)$. In addition, the $F_{n}(z)$ are found to be a family of solutions to a class of second-order hypergeometric differential equations when $n \equiv 0(\bmod m)$. We also found that the zeros of $F_{n}(z)$ when $n \equiv 0(\bmod m)$, or $n \equiv \frac{m}{2}(\bmod m)$ if $m$ is even, are located on $S_{m}$. The asymptotic behavior of the zeros of $F_{n}(z)$ is also determined explicitly. Numerical results for the zeros of $F_{n}(z)$ will be given in $\S 3$.

\section{MAIN RESULTS AND PROOFS}

As we shall see, the Faber polynomials for $S_{m}$ with $m>2$ enjoy certain properties that are similar to those satisfied by Chebyshev polynomials. Our main results in this direction are the following: 
Theorem. Let $F_{n}(z)$ be the Faber polynomials of $S_{m}$ of degree $n$. Then

(i) For $n \geq 1$,

$$
F_{n}(z)=\frac{2 n}{m} \sum_{j=0}^{\left[\frac{n}{m}\right]}(-1)^{j} \frac{\Gamma\left(\frac{2 n}{m}-j\right)}{\Gamma\left(\frac{2 n}{m}-2 j+1\right) j !} z^{n-m j},
$$

where

$$
\left[\frac{n}{m}\right]= \begin{cases}\frac{n}{m} & \text { if } n \equiv 0(\bmod m), \\ \frac{n-k}{m} & \text { if } n \equiv k(\bmod m), k=0,1,2, \ldots, m-1 .\end{cases}
$$

(ii) If $n \equiv 0(\bmod m)$, then $F_{n}(z)$ satisfies the following differential equation:

$$
\left(4-z^{m}\right) z y^{\prime \prime}-\left[z^{m}+2 m-4\right] y^{\prime}+n^{2} z^{m-1} y=0 .
$$

(iii) If $n \equiv 0(\bmod m)$, or $n \equiv \frac{m}{2}(\bmod m)$ when $m$ is even, then the zeros of $F_{n}(z)$ are located on $S_{m}$.

(iv) For $n \geq 1$, the zero distribution of $F_{n}(z)$ for $S_{m}$ is given by

$$
d \mu(t)=\frac{1}{\pi} \frac{t^{(m-2) / 2}}{\sqrt{4-t^{m}}} d t, \quad t \in S_{m} .
$$

One can see that the above theorem generalizes (1.4), (1.5), and (1.6) when $m=2$. We now proceed to prove our theorem.

Proof of (i). It is known [3, p. 395] that for $m=2,3, \ldots$,

$$
z=\Psi(w)=w\left(1+\frac{1}{w^{m}}\right)^{2 / m}
$$

maps $|w|>1$ conformally onto $\mathbf{C}^{*} \backslash S_{m}$. Let $\Phi(z)$ be its inverse. It follows from the definition of Faber polynomials that $F_{n}(z)$ generated by $S_{m}$ is given by

$$
F_{n}(z)=\sum_{j=0}^{n} c_{n-j} z^{n-j},
$$

where $c_{n-j}$ is the Laurent coefficient in the expansion of $\{\Phi(z)\}^{n}$, that is, for $j=0,2,3, \ldots, n$,

$$
c_{n-j}=\int_{|z|=R} \frac{\{\Phi(z)\}^{n}}{z^{n-j+1}} d z,
$$

with $R$ chosen sufficiently large so that $S_{m}$ is contained in the interior of the region bounded by the circle $|z|=R$. Alternatively, using the substitution $z=\Psi(w)$, we obtain for $j=0,2,3, \ldots, n$,

$$
c_{n-j}=\int_{|w|=r>\rho} \frac{w^{n} \Psi^{\prime}(w)}{\{\Psi(w)\}^{n-j+1}} d w .
$$

By the symmetry of $S_{m}$, we see that $\Psi(w)$ is an $m$-fold symmetric mapping function (i.e., $\left.\Psi\left(\exp \left(\frac{2 k \pi i}{m} w\right)\right)=\exp \left(\frac{2 k \pi i}{m}\right) \Psi(w), k=0,1, \ldots, m-1\right)$. It is easy to see that $c_{n-j}=0$ if $j \neq 0(\bmod m)$. Thus, $F_{n}(z)$ has the following form:

$$
F_{n}(z)=\sum_{j=0}^{\left[\frac{n}{m}\right]} c_{n-m j} z^{n-m j}
$$


By Cauchy's Theorem, we see that the coefficients $c_{n-m j}$ are the same as those of $\frac{1}{w}$ in the expansion of $w^{n} \Psi^{\prime}(w) /[\Psi(w)]^{n-m j+1}$. Using (2.4), we get

$$
\Psi^{\prime}(w)=\left(1-\frac{1}{w^{m}}\right)\left(1+\frac{1}{w^{m}}\right)^{(2-m) / m} .
$$

Thus,

$$
\begin{aligned}
\frac{w^{n} \Psi^{\prime}(w)}{[\Psi(w)]^{n-m j+1}}= & w^{m j-1}\left(1-\frac{1}{w^{m}}\right)\left(1+\frac{1}{w^{m}}\right)^{-(m+2 n-2 m j) / m} \\
= & w^{m j-1}\left(1+\frac{1}{w^{m}}\right)^{-(m+2 n-2 m j) / m} \\
& -w^{m j-m-1}\left(1+\frac{1}{w^{m}}\right)^{-(m+2 n-2 m j) / m}
\end{aligned}
$$

Noticing that $|w|>1$, we have

$$
\left(1+\frac{1}{w^{m}}\right)^{-(m+2 n-2 m j) / m}=\sum_{i=0}^{\infty}(-1)^{i}\left(\frac{m+2 n-2 m j}{m}\right)_{i} \frac{w^{-m i}}{i !},
$$

where

$$
(\alpha)_{i}=\alpha(\alpha+1)(\alpha+2) \cdots(\alpha+i-1), \quad(\alpha)_{0}=1, \quad(\alpha)_{-1}=0 .
$$

Thus, the coefficient of $\frac{1}{w}$ is given by

$$
\begin{aligned}
c_{n-m j} & =(-1)^{j} \frac{\left(\frac{m+2 n-2 m j}{m}\right)_{j}}{j !}-(-1)^{j-1} \frac{\left(\frac{m+2 n-2 m j}{n}\right)_{j-1}}{(j-1) !} \\
& =(-1)^{j} \frac{2 m}{n} \frac{\left(\frac{2 n}{m}+1-2 j\right)\left(\frac{2 n}{m}+1-2 j+1\right) \cdots\left(\frac{2 n}{m}+1-2 j+j-2\right)}{j !} \\
& =(-1)^{j} \frac{2 n}{m} \frac{\Gamma\left(\frac{2 n}{m}-j\right)}{\Gamma\left(\frac{2 n}{m}-2 j+1\right) j !} \cdot \square
\end{aligned}
$$

Proof of (ii). Let $x=z^{m}$ in (2.2). Then we have the following hypergeometric differential equation:

$$
x(4-x) y^{\prime \prime}+(2-x) y^{\prime}+n^{2} y=0 .
$$

Replacing $n$ by $n m$ in (2.1) and substituting $z^{m}$ for $x$, we get

$$
y:=F_{n m}(z)=2 n \sum_{j=0}^{n}(-1)^{j} \frac{\Gamma(2 n-j)}{\Gamma(2 n-2 j+1) j !} x^{n-j} .
$$

It is easy to verify that $y$ is the polynomial solution of $(2.5)$. Therefore, $F_{n m}(z)$ is the polynomial solution of (2.2).

Proof of (iii). If $n \equiv 0(\bmod m)$, or $n \equiv \frac{m}{2}(\bmod m) \quad(m$ is even), then by (1.4)

$$
\begin{gathered}
F_{n m}(z)=2 n \sum_{j=0}^{n}(-1)^{j} \frac{\Gamma(2 n-j)}{\Gamma(2 n-2 j+1) j !} z^{n m-m j} \\
F_{n m+m / 2}(z)=(2 n+1) z^{m / 2} \sum_{j=0}^{n}(-1)^{j} \frac{\Gamma(2 n+1-j)}{\Gamma(2 n+1-2 j+1) j !} z^{n m-m j}
\end{gathered}
$$


Comparing $F_{m n}(z)$ and $F_{m n+m / 2}(z)$ with (1.4) yields

$$
F_{m n}(z)=T_{2 n}\left(z^{m / 2}\right), \quad F_{m n+m / 2}(z)=T_{2 n+1}\left(z^{m / 2}\right) .
$$

Since the zeros of $T_{2 n}(x)$ and $T_{2 n+1}(x)$ are located on [-2, 2], it follows from (2.6) that the zeros of $F_{m n}(z)$ and $F_{m n+m / 2}(z)$ are located on $S_{m}$.

Remark. Using (2.4), we can derive the relations

$$
\Phi(z)=\left\{\phi\left(z^{m / 2}\right)\right\}^{2 / m} \text { and } \Psi(z)=\left\{\psi\left(z^{m / 2}\right)\right\}^{2 / m},
$$

where $\psi(w)=w+\frac{1}{w}$ and $\phi(z)$ is the inverse mapping function of $\psi(w)$. By the above equations and the definition of the Faber polynomials, one can also obtain (2.6).

Proof of (iv). In order to prove (iv), we first develop some notation. The term "capacity" means inner logarithmic capacity (cf. [8, p. 55]). For any set $E \subset \mathbf{C}$, the capacity of $E$ will be denoted by $C(E)$. If $E$ is a compact set with positive capacity, then $\mu_{E}$ shall denote the unique unit equilibrium measure on $E$ with the property that

$$
\int_{E} \log |x-t| d \mu_{E}(t)=\log C(E)
$$

quasi-everywhere (q.e.) on $E$ (cf. [8, p. 60]). A property is said to hold q.e. on a set $A$ if the subset $E$ of $A$ where it does not hold satisfies $C(E)=0$.

To each $p_{n}(z)=\prod_{k=1}^{n}\left(z-z_{k}\right)$, we associate the normalized zero distribution measure $\nu\left(p_{n}\right)$ defined by

$$
\nu\left(p_{n}\right):=\frac{1}{n} \sum_{k=1}^{n} \delta_{z_{k}},
$$

where $\delta_{z_{k}}$ is the point distribution with total mass 1 at $z_{k}$.

We now prove the following lemma.

Lemma. Let $E$ be a compact subset (not a single point) whose complement $\mathbf{C}^{*} \backslash E$ is simply connected. Assume that $E$ has empty interior. Then the asymptotic distribution of zeros of Faber polynomials $F_{n}(z)$ coincides with the equilibrium measure of the set $E$. More precisely, the normalized zero distribution measures $\nu\left(F_{n}\right)$ converge in the weak-star topology to $\mu_{E}$.

Proof. By Theorem 2.3 in [6], we need only to prove that Faber polynomials are an asymptotically minimal norm sequence of monic polynomials, that is,

$$
\limsup _{n \rightarrow \infty}\left\|F_{n}\right\|_{\operatorname{supp}\left(\mu_{E}\right)}^{1 / n} \leq \operatorname{cap}(E)=1,
$$

where $\mu_{E}$ is the equilibrium distribution of $E$. It is known from [5, p. 108] that for $r>1$,

$$
\frac{1}{2}|\Phi(z)|^{n} \leq\left|F_{n}(z)\right| \leq \frac{3}{2}|\Phi(z)|^{n},
$$

where $z$ is on or exterior to $C_{r}=\{|\Phi(z)|=r\}$. For $n$ large enough we know that

$$
\left\|F_{n}\right\|_{E} \leq\left\|F_{n}\right\|_{C_{r}} \leq \frac{3}{2} r^{n}
$$

Taking the $n$th roots in (2.8), we get $\left\|F_{n}\right\|_{E}^{1 / n} \leq\left(\frac{3}{2}\right)^{1 / n} r$, so we have

$$
\limsup _{n \rightarrow \infty}\left\|F_{n}\right\|_{E}^{1 / n} \leq r \text {. }
$$

Letting $r \rightarrow 1$, we get (2.7). 
We now go back to prove (iv). Clearly, the $m$-star has empty interior. According to our lemma, we know that the limiting distribution of the zeros of Faber polynomials associated with $S_{m}$ coincides with equilibrium measure $d \mu^{*}(t)$ for $S_{m}$. We now determine $d \mu^{*}(t)$. Define

$$
M_{k}:=\int_{S_{m}} t^{k} d \mu^{*}(t), \quad k=0,1, \ldots,
$$

where $M_{k}$ is the so-called $k$ th moment of the equilibrium distribution on $S_{m}$. On the other hand, $M_{k}$ can also be expressed as (cf. [4, p. 345])

$$
M_{k}=\frac{1}{\pi i} \int_{|s|=r}[\Psi(s)]^{k} \frac{d s}{s}
$$

for $r>1$, where $z=\Psi(w)=w\left(1+1 / w^{m}\right)^{2 / m}$ maps the exterior of $|w|=1$ onto the exterior of $S_{m}$ (cf. [3, p. 395]).

We now compute the $k$ th moment $M_{k}$ from (2.10). Let

$$
t=\Psi(s)=s\left(1+\frac{1}{s^{m}}\right)^{2 / m}=\left(s^{m / 2}+s^{-m / 2}\right)^{2 / m}
$$

in $(2.10)$. Then

$$
\begin{aligned}
d t & =\left(s^{m / 2}+s^{-m / 2}\right)^{2 / m-1}\left(s^{m / 2-1}-s^{-m / 2-1}\right) d s \\
& =\left(s^{m / 2}+s^{-m / 2}\right)^{-(m-2) / m}\left(s^{m / 2}-s^{-m / 2}\right) \frac{d s}{s}=t^{1-m / 2} i \sqrt{4-t^{m}} \frac{d s}{s},
\end{aligned}
$$

and so

$$
M_{k}=\frac{1}{\pi i} \int_{|s|=r}[\Psi(s)]^{k} \frac{d s}{s}=\frac{1}{\pi} \int_{C_{r}} t^{k} \sqrt{\frac{t^{m-2}}{4-t^{m}}} d t,
$$

where $C_{r}$ is the image of $|w|=r$ under the mapping $\Psi(w)$.

Letting $r \rightarrow 1$, we get

$$
M_{k}=\frac{1}{\pi} \int_{S_{m}} t^{k} \sqrt{\frac{t^{m-2}}{4-t^{m}}} d t
$$

By (2.9) we have

$$
\int_{S_{m}} t^{k} d \mu^{*}(t)=M_{k}=\frac{1}{\pi} \int_{S_{m}} t^{k} \sqrt{\frac{t^{m-2}}{4-t^{m}}} d t, \quad k=0,1, \ldots
$$

Thus, $d \mu^{*}(t)=d \mu(t)$, where $d \mu(t)$ is defined in (2.3).

We have completed the proof of the theorem.

The situation for obtaining the locations of the zeros of $F_{n}(z)$ for every $n$ is not so favorable. However, supported by (iii) in our theorem, and numerical experiments, we formulate the following.

Conjecture. The zeros of Faber polynomials generated by the regular $m$-star $S_{m}$ $(m=3,4, \ldots)$ are located on $S_{m}$ for every $n \geq 1$.

For a sequence of polynomials $F_{n m}(z)$, and $F_{n m+m / 2}(z)$ when $m$ is even, our theorem confirmed this conjecture. For other sequences of Faber polynomials, 
we provide in $\S 3$ numerical results produced by Matlab in the cases when $m=$ 3,4 , and 5 .

\section{NUMERICAL RESULTS ON THE ZEROS OF FABER POLYNOMIALS}

We first briefly state our algorithm for computing the zeros of Faber polynomials generated by a set $E$.

Let

$$
\Psi(z)=w+b_{0}+\frac{b_{1}}{w}+\frac{b_{2}}{w^{2}}+\cdots
$$

be the mapping function as in (1.2) associated with a set $E$. Then

$$
\frac{\Psi^{\prime}(w)}{\Psi(w)-z}=\sum_{n=0}^{\infty} \frac{F_{n}(z)}{w^{n+1}}, \quad|w|>\rho_{E}, \quad z \in E .
$$

It follows from (3.2) that $F_{n}(z)$ satisfies the recurrence relation

$$
F_{n+1}(z)=\left(z-b_{0}\right) F_{n}(z)-\sum_{k=1}^{n-1} b_{k} F_{n-k}(z)-(n+1) b_{n},
$$

with initial condition $F_{0}(z)=1$. This difference equation for Faber polynomials plays a very important role in computing the zeros of $F_{n}(z)$. Our algorithm consists of four steps:

Step 1. Determine the coefficients $b_{k}$ 's of the mapping function $\Psi(w)$ as in (3.1).

Step 2. Compute the coefficients of $F_{n}(z)$ by using (3.3).

Step 3. Find the zeros of $F_{n}(z)$.

Step 4. Plot the zeros in the complex plane.

We now apply the algorithm to compute the zeros of $F_{n}(z)$ for $S_{m}$. The results are shown in Figures 1-6 (see pp. 284-286).

For $m=2,3, \ldots$, we normalize the mapping function such that

$$
\Psi(w)=w\left(1+\frac{1}{w^{m}}\right)^{2 / m} .
$$

Then

$$
\Psi(w)=w+\sum_{k=1}^{\infty} \frac{b_{m k-1}}{w^{m k-1}}
$$

where

$$
b_{m k-1}=(-1)^{k-1} \frac{2(m-2)(2 m-2) \cdots((k-1) m-2)}{m^{k} k !} .
$$

By the recurrence relation (3.3), we find, in particular for $m=3$,

$$
\begin{aligned}
& F_{0}(z)=1, \quad F_{1}(z)=z, \quad F_{2}(z)=z^{2}, \\
& F_{3}(z)=z^{3}-2, \quad F_{4}(z)=z^{4}-\frac{8}{3} z, \quad F_{5}(z)=z^{5}-\frac{10}{3} z^{2}, \\
& F_{6}(z)=z^{6}-4 z^{3}+2, \quad F_{7}(z)=z^{7}-\frac{14}{3} z^{4}+\frac{35}{9} z, \quad F_{8}(z)=z^{8}-\frac{16}{3} z^{5}+\frac{56}{9} z^{2} . \\
& F_{9}(z)=z^{9}-6 z^{6}+z^{3}-2, \quad F_{10}(z)=z^{10}-\frac{20}{3} z^{7}+\frac{110}{9} z^{4}-\frac{400}{81} z .
\end{aligned}
$$




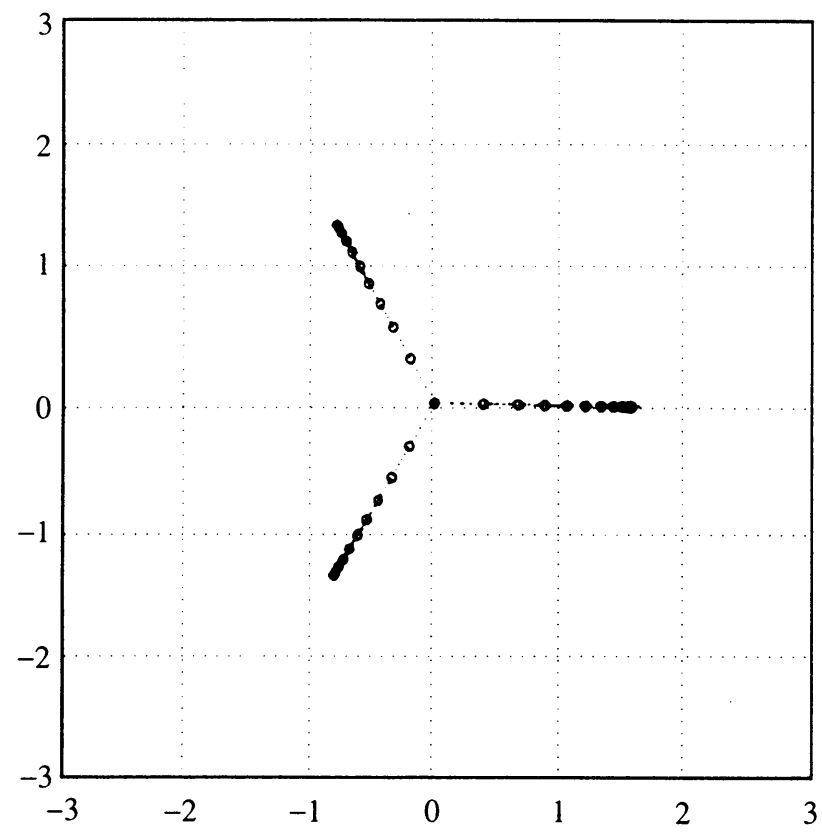

Figure 1. Zeros of $F_{n}(z)$ for 3-star when $n=31$

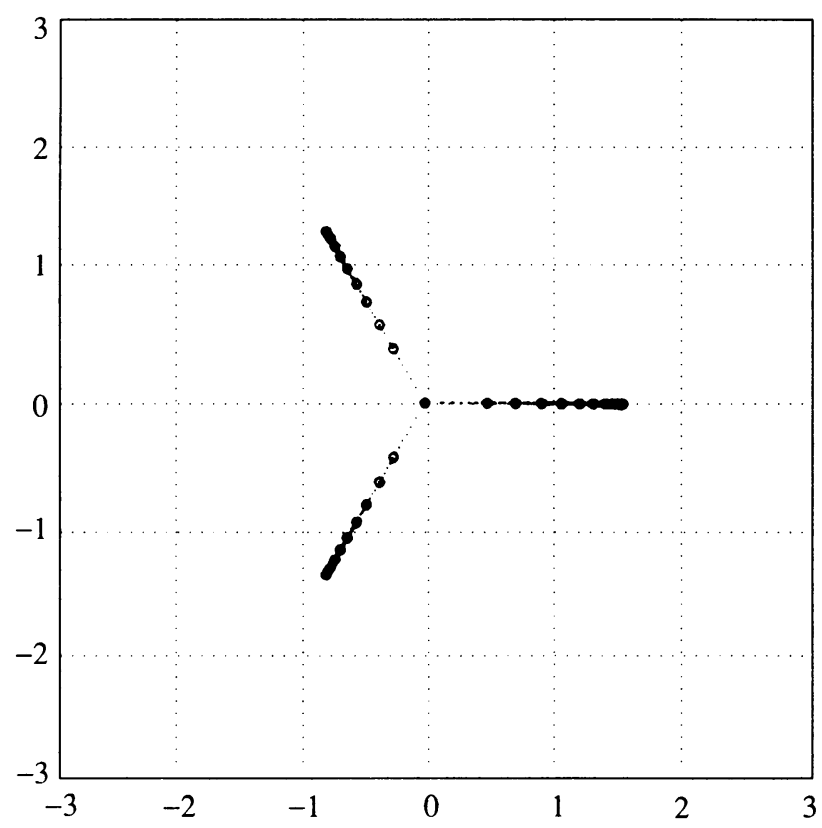

Figure 2. Zeros of $F_{n}(z)$ for 3-star when $n=32$ 


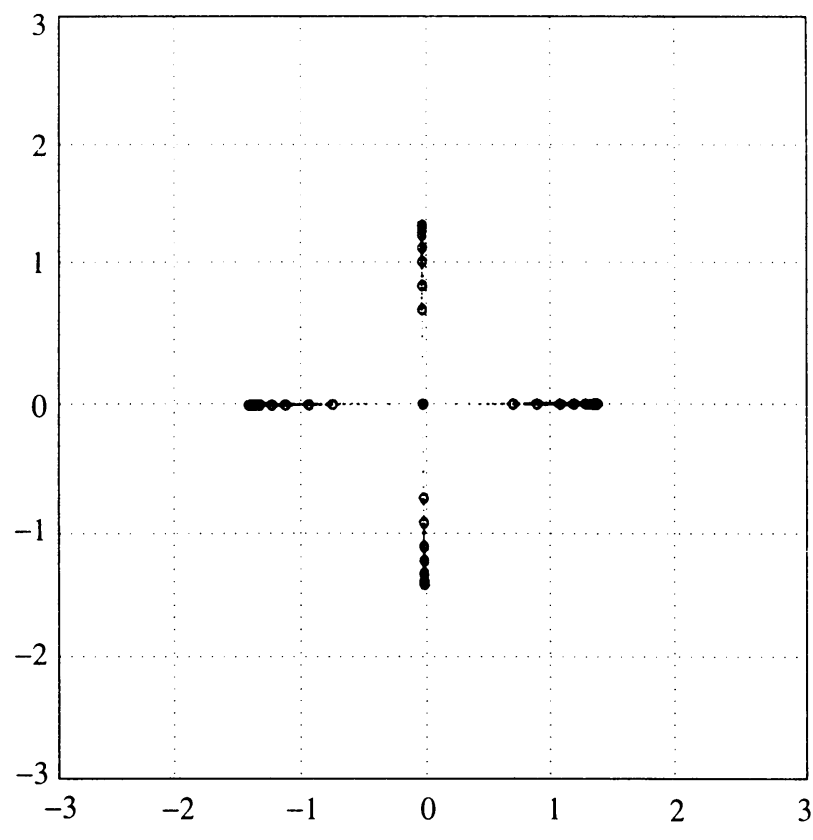

FIGURE 3. Zeros of $F_{n}(z)$ for 4-star when $n=31$

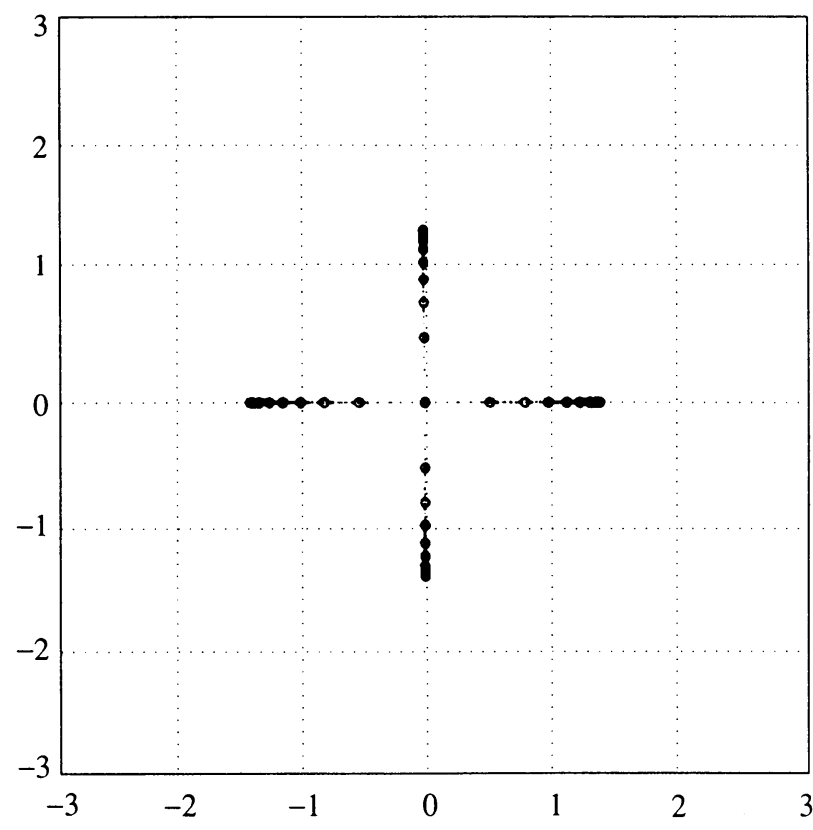

FigURE 4. Zeros of $F_{n}(z)$ for 4-star when $n=33$ 


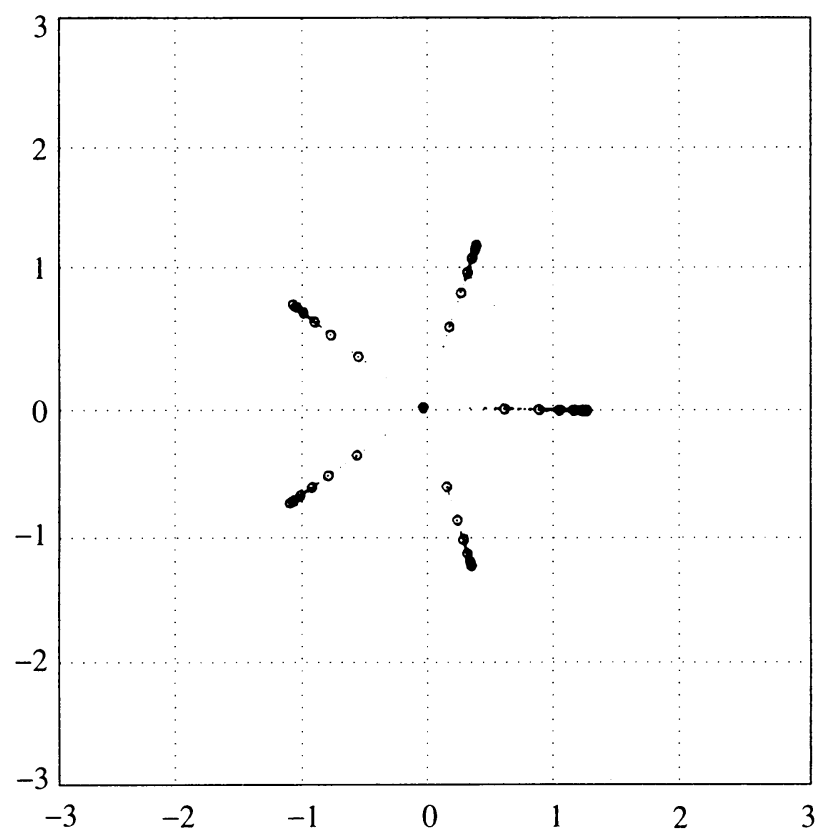

Figure 5. Zeros of $F_{n}(z)$ for 5-star when $n=31$

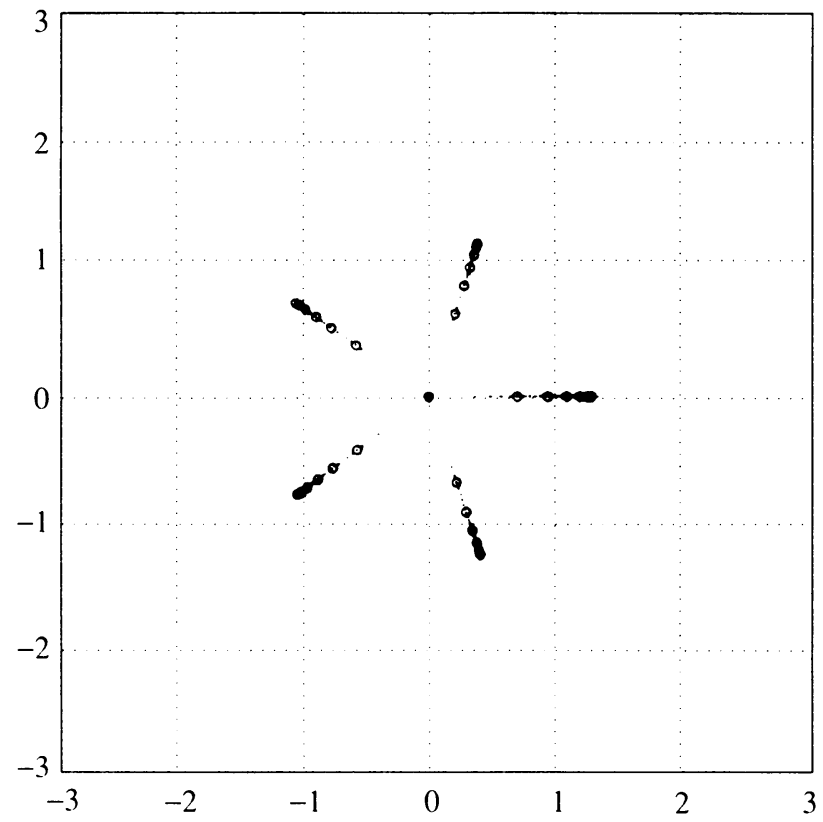

Figure 6. Zeros of $F_{n}(z)$ for 5-star when $n=32$ 


\section{BIBLIOGRAPHY}

1. J. H. Curtiss, Faber polynomials and the Faber series, Amer. Math. Monthly 78 (1971), 577-596.

2. G. Faber, Über Polynomische Entwickelungen, Math. Ann. 57 (1903), 389-408.

3. P. Henrici, Applied and computational complex analysis, Vol. I, Wiley, New York, 1974.

4. E. Hille, Analytic function theory (Introduction to higher mathematics), Vol. II, Ginn, Boston, 1962.

5. A. I. Markushevich, Theory of analytic function, Vol. 3, Prentice-Hall, Englewood Cliffs, NJ, 1967.

6. H. N. Mhaskar and E. B. Saff, The distribution of zeros of asymptotically extremal polynomials, J. Approx. Theory 65 (1991), 279-300.

7. G. Szegö, Orthogonal polynomials, 4th ed., Amer. Math Soc., Providence, RI, 1975.

8. M. Tsuji, Potential theory in modern function theory, Maruzen, Tokyo, 1959.

Department of Mathematics, Nova University, Ft. Lauderdale, Florida 33314

E-mail address, M. He: hem@polaris.nova.edu 